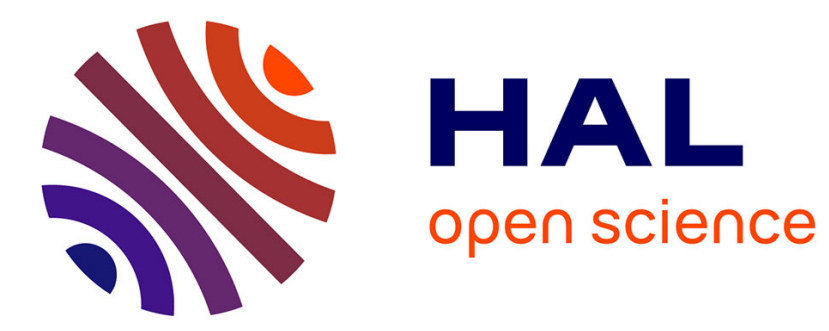

\title{
Revisiting the radiative impact of dust on Mars using the LMD Global Climate Model
}

\author{
J.-B. Madeleine, F. Forget, E. Millour, L. Montabone, M.J. Wolff
}

\section{To cite this version:}

J.-B. Madeleine, F. Forget, E. Millour, L. Montabone, M.J. Wolff. Revisiting the radiative impact of dust on Mars using the LMD Global Climate Model. Journal of Geophysical Research. Planets, 2011, 116 (11), pp.E11010. 10.1029/2011JE003855 . hal-01120102

\section{HAL Id: hal-01120102 \\ https://hal.science/hal-01120102}

Submitted on 24 Feb 2015

HAL is a multi-disciplinary open access archive for the deposit and dissemination of scientific research documents, whether they are published or not. The documents may come from teaching and research institutions in France or abroad, or from public or private research centers.
L'archive ouverte pluridisciplinaire HAL, est destinée au dépôt et à la diffusion de documents scientifiques de niveau recherche, publiés ou non, émanant des établissements d'enseignement et de recherche français ou étrangers, des laboratoires publics ou privés. 


\title{
Revisiting the radiative impact of dust on Mars using the LMD Global Climate Model
}

\author{
J.-B. Madeleine, ${ }^{1,2}$ F. Forget, ${ }^{1}$ E. Millour, ${ }^{1}$ L. Montabone, ${ }^{1}$ and M. J. Wolff ${ }^{3}$ \\ Received 3 May 2011; revised 1 September 2011; accepted 1 September 2011; published 22 November 2011.
}

[1] Airborne dust is the main driver of Martian atmospheric temperature, and accurately accounting for its radiative effect in Global Climate Models (GCMs) is essential. This requires the modeling of the dust distribution and radiative properties, and when trying to simulate the true climate variability, the use of the observed dust column opacity to guide the model. A recurrent problem has been the inability of Mars GCMs to predict realistic temperatures while using both the observed dust radiative properties and column opacity. One would have to drive the model with a tuned opacity to reach an agreement with the observations, thereby losing its self-consistency. In this paper, we show that using the most recently derived dust radiative properties in the LMD (Laboratoire de Météorologie Dynamique) GCM solves this problem, which was mainly due to the underestimation of the dust single scattering albedo in the solar domain.

However, an overall warm temperature bias remains above the $1 \mathrm{hPa}$ pressure level. We therefore refine the model by implementing a "semi-interactive" dust transport scheme which is coupled to the radiative transfer calculations. This scheme allows a better representation of the dust layer depth in the model and thereby removes the remaining warm bias. The LMD/GCM is now able to predict accurate temperatures without any tuning of the dust opacity used to guide the model. Remaining discrepancies are discussed, and seem to be primarily due to the neglect of the radiative effect of water-ice clouds, and secondarily to persisting uncertainties in the dust spatial distribution.

Citation: Madeleine, J.-B., F. Forget, E. Millour, L. Montabone, and M. J. Wolff (2011), Revisiting the radiative impact of dust on Mars using the LMD Global Climate Model, J. Geophys. Res., 116, E11010, doi:10.1029/2011JE003855.

\section{Introduction}

[2] About 40 years ago, Gierasch and Goody [1972] demonstrated that the thermal structure of the Martian atmosphere cannot be accounted for by a purely gaseous $\mathrm{CO}_{2}$ atmosphere, without the contribution of atmospheric dust. Dust is, indeed, the main driver of the Martian climate, and its radiative properties (extinction efficiency $\mathrm{Q}_{\mathrm{ext}}$, single scattering albedo $\omega_{0}$ and asymmetry parameter $g$ ) have to be known in detail to accurately predict the heating rates and temperatures in a Mars Global Climate Model (GCM).

[3] The most appropriate way to simulate the details of the present climate is to drive the GCM with observation-derived dust opacities. However, it has been difficult in the past to obtain realistic temperatures by using the observed dust column opacity. The latter had to be tuned to reach reasonable temperatures, raising some doubts on either GCMs or dust radiative properties used to compute the heating rates.

\footnotetext{
${ }^{1}$ Laboratoire de Météorologie Dynamique, CNRS/UPMC/IPSL, Paris, France.

${ }^{2}$ Now at Department of Geological Sciences, Brown University, Providence, Rhode Island, USA.

${ }^{3}$ Space Science Institute, Boulder, Colorado, USA.

Copyright 2011 by the American Geophysical Union. 0148-0227/11/2011JE003855
}

[4] The dust radiative properties are difficult to retrieve, and are associated with many uncertainties to which GCMs are extremely sensitive. Heating rates are proportional to (1 $\left.\omega_{0}\right)$ in the optically thin limit, and an uncertainty of $5 \%$ for a single scattering albedo $\omega_{0}$ of about 0.9 in the solar domain corresponds to an error on the heating rate of about 50\% [Forget et al., 1999]. The asymmetry factor $g$ is also essential, because a decrease in $g$ at solar wavelengths corresponds to a increase in backscattering and hence in the amount of sunlight deposited within the atmosphere [Pollack et al., 1995]. Moreover, the same decrease in $g$ reduces the amount of solar radiation that reaches the surface and impacts on the surface temperature and greenhouse effect [Wilson and Smith, 2006].

[5] Thanks to the numerous new missions of the last decade, many improvements have been made in our knowledge of dust radiative properties, ultimately leading to the retrieval of the fundamental refractive index, both in the visible and infrared regions [Wolff et al., 2006, 2009]. This allows us to compute the single scattering parameters for dust particles of different sizes, which is essential to fully account for their effect on GCM temperatures. The goal of this paper is to find the best way to use this new data set, in order to create a self-consistent climate model, i.e. a model in 
Table 1. Main Characteristics of the Three GCM Experiments

\begin{tabular}{|c|c|c|c|c|}
\hline \multirow[b]{2}{*}{ Simulation } & \multicolumn{3}{|c|}{ Dust Single Scattering Properties } & \multirow[b]{2}{*}{ Opacity Profiles $^{\mathrm{a}}$} \\
\hline & Data Set & Spatial Variation & Computation Method & \\
\hline 1 & $\begin{array}{l}\text { Single scattering parameters } \\
\text { of Ockert-Bell et al. [1997] } \\
\text { and Forget [1998] }\end{array}$ & Spatially constant & $\begin{array}{l}\text { Direct merging of the two data sets } \\
\quad \text { using } \tau_{0.67 \mu \mathrm{m}} / \tau_{9 \mu \mathrm{m}}=2\end{array}$ & $\begin{array}{l}\text { Analytical function } \\
\text { Modified Conrath profile }\end{array}$ \\
\hline 2 & $\begin{array}{l}\text { Refractive index } m=n+i k \\
\text { of Wolff et al. [2006, 2009] }\end{array}$ & Spatially constant & $\begin{array}{l}\text { T-Matrix generated using } m \\
\text { Gamma dist., }{ }^{b} \mathrm{r}_{\text {eff }}=1.5 \mu \mathrm{m}, \nu_{\mathrm{eff}}=0.3\end{array}$ & $\begin{array}{l}\text { Analytical function } \\
\text { Modified Conrath profile }\end{array}$ \\
\hline 3 & $\begin{array}{l}\text { Refractive index } m=n+i k \\
\text { of Wolff et al. [2006, 2009] }\end{array}$ & $\begin{array}{l}\text { Space-varying properties } \\
\text { based on predicted sizes }\end{array}$ & $\begin{array}{l}\text { T-Matrix generated using } m \\
\text { Log-normal dist., }{ }^{\mathrm{b}} \text { variable } \mathrm{r}_{\text {eff }}, \nu_{\text {eff }}=0.3\end{array}$ & $\begin{array}{l}\text { Model predicted } \\
\text { Two-moment scheme }\end{array}$ \\
\hline
\end{tabular}

${ }^{a}$ Opacity profiles are always linearly scaled to match the column dust opacity measured by TES.

${ }^{\mathrm{b}}$ Randomly oriented oblate cylinder with an axial ratio of 1 .

which temperatures and dust opacities are both consistent with observations.

[6] For this, the following questions will be addressed:

[7] 1. What are the effects of the new dust radiative properties on the LMD/GCM?

[8] 2. What is the impact of the dust layer properties (thickness and particle sizes) on the simulated temperatures?

[9] 3. What can we learn from radiatively active dust experiments about the spatial distribution and size of the dust particles?

[10] To answer these questions, we make extensive use of the TES (Thermal Emission Spectrometer onboard Mars Global Surveyor) measurements to constrain our model, as well as to compare the simulated temperatures with the retrieved profiles. TES is a thermal infrared spectrometer $(5.8-50 \mu \mathrm{m})$ which also includes two broadband visible/ near-IR $(0.3-2.9 \mu \mathrm{m})$ and thermal $(5.1-150 \mu \mathrm{m})$ channels [Christensen et al., 2001]. We use in this paper the retrievals of atmospheric temperature (using the $15 \mu \mathrm{m} \mathrm{CO}_{2}$ band), dust column opacity $(9.3 \mu \mathrm{m})$ and water-ice cloud column opacity $(12.1 \mu \mathrm{m})$, which are described by Conrath et al. [2000], Smith et al. [2000] and Pearl et al. [2001], respectively. The MGS mapping mission covers Mars Years 2427. Martian years are defined by Clancy et al. [2000], and the first Martian year begins on April 11, 1955.

[11] Three simulations are carried out, and the corresponding model configurations are described in section 2 . Temperatures predicted by the LMD/GCM when using the dust radiative properties of Ockert-Bell et al. [1997] and Forget [1998] on the one hand, and the more recent optical indices of Wolff et al. [2006, 2009] on the other, are analyzed in section 3 and compared to the TES temperature measurements [Smith, 2004]. These two simulations are later referred to as case 1 and case 2 simulations. The GCM radiative scheme is then connected to a dust transport model, which computes the dust spatial distribution and particle size. The predicted spatial distribution is used to compute the $3 \mathrm{D}$ opacity field, and each opacity profile is then multiplied by a constant to match the dust column opacity observed by TES [Smith, 2004]. The particle sizes are also used to compute spatially and temporally variable radiative properties. This simulation is referred to as case 3 simulation, and is analyzed in section 4. Both Martian years 25 and 26 are simulated, to evaluate the model under the relatively clear conditions of MY26, and the dusty conditions of MY25, during which the 2001 global dust storm occurred. It is worth noting that MY25 and MY26 are actually quite similar for the bulk of the annual cycle, with major differences occurring between $\mathrm{L}_{\mathrm{s}}=180^{\circ}$ and $\mathrm{L}_{\mathrm{s}}=260^{\circ}$. The radiative effect of water-ice clouds is not included in the model to focus on dust alone, and will be the subject of another article in the near future.

\section{Modeling Approach}

[12] The three simulations described in this paper are summarized in Table 1 . They have a resolution of $5.625 \times$ $3.75^{\circ}$ in the horizontal, and 25 levels in the vertical, from the ground to $\sim 100 \mathrm{~km}$. The two first simulations use a modified Conrath profile (see the next section and equation (2) for further details) to describe the vertical distribution of dust (as was the case in the work of Forget et al. [1999]) and spatially uniform radiative properties. The last simulation uses interactive dust profiles and varying radiative properties, based on a dust transport model which predicts the shape of the dust vertical profile and the size of the dust particles. Each of the three simulations is further described below.

\subsection{Ockert-Bell et al. Model (Case 1 Simulation)}

[13] The dust layer is characterized by the amount and spatial distribution of dust, as well as the radiative properties of the dust particles. The amount of dust in the atmosphere is indirectly given by its dust optical depth:

$$
\mathrm{d} \tau_{\lambda}=\frac{3}{4} \frac{Q_{\text {ext }, \lambda} q}{\rho_{p} r_{\text {eff }} g} \mathrm{~d} p
$$

where $\mathrm{Q}_{\text {ext }}$ is the dust extinction efficiency, $q$ the mass mixing ratio, $\rho_{p}$ the dust particle density $\left(2500 \mathrm{~kg} \mathrm{~m}^{-3}\right)$, and $\mathrm{r}_{\mathrm{eff}}$ the effective radius. In the case 1 and case 2 simulations, we assume a homogeneous size and extinction efficiency of the dust particles. Consequently, the opacity in each layer is directly proportional to the amount of dust $q$ and the pressure differential $\mathrm{d} p$. Since dust is not carried explicitly by the model in these simulations, a modified Conrath vertical profile is assumed [Conrath, 1975; Forget et al., 1999], and the dust opacity differential obeys the relation:

$$
\mathrm{d} \tau_{\lambda}(p) \propto \mathrm{d} p \exp \left\{0.007\left[1-\left(\frac{p_{\text {ref }}}{p}\right)^{70 / z_{\max }}\right]\right\},
$$

where $p<p_{\text {ref, }}$, with $p_{\text {ref }}$ the reference pressure $(6.1 \mathrm{hPa})$. When the atmospheric pressure $p$ is larger than $p_{\text {ref }}$, the dust 
opacity is assumed constant. The dust layer depth $z_{\max }$ is given by an analytical function that fits the measurements of $z_{\max }$ achieved by Jaquin et al. [1986] (see Montmessin et al. [2004, section 2.1] for further information). The variation of this function at the equator is represented in Figure 5c (black sinusoid). The variations in $z_{\max }$ are identical from one Martian year to another, and cannot capture the interannual variability of the dust layer thickness. The opacity profile given by equation (2) is then linearly scaled so that the dust column opacity in the model matches the observed TES column opacity at $9.3 \mu \mathrm{m}$ (see Figure 5b and Smith [2004]). It is worth remembering that TES column opacity is an absorption opacity, and it has to be converted to an extinction opacity, which is the opacity actually needed by the GCM. As discussed in detail by Wolff and Clancy [2003, section 7.2.1], this conversion can be done without large error using a factor of 1.3 , assuming the canonical $r_{\text {eff,dust }}=1.5 \mu \mathrm{m}$ (see section 2.2 and Wolff et al. [2009]). Consequently, $\tau_{\mathrm{GCM}}(9.3 \mu \mathrm{m})=$ $\tau_{\text {TES }}(9.3 \mu \mathrm{m}) \times 1.3$, and the GCM is constrained by the observed and untuned dust column opacity. This is true for all the simulations presented in this paper.

[14] Once the opacity in each layer is known, the model needs the dust single scattering parameters, which will be used by the radiative transfer scheme. The radiative transfer codes at solar wavelengths and outside the $15 \mu \mathrm{m} \mathrm{CO}_{2}$ band are both based on the two stream algorithm of Toon et al. [1989]. Their channels include two solar bands $(0.1-0.5 \mu \mathrm{m}$ and $0.5-5 \mu \mathrm{m})$, the silicate band $(5-11.5 \mu \mathrm{m})$, and the rest of the IR domain (20-200 $\mu \mathrm{m})$. The net exchange formulation [Dufresne et al., 2005] is used in the $15 \mu \mathrm{m} \mathrm{CO}_{2}$ band (11.5$20 \mu \mathrm{m})$, where dust scattering is neglected [Forget et al., 1999, section 4.2.2]. In this band, only absorption by dust is taken into account, and added to that of $\mathrm{CO}_{2}$ by using $Q_{\mathrm{abs}}=$ $Q_{\text {ext }}\left(1-\omega_{0}\right)$ [Forget et al., 1999; Wolff and Clancy, 2003].

[15] Providing dust radiative properties covering the entire solar and thermal infrared spectral range for climate modeling required long-term efforts. Several data sets have been available since Mariner 9 in 1972. The first general circulation models including a comprehensive radiative transfer model [Pollack et al., 1990; Haberle et al., 1993, 1997, 1999; Hourdin et al., 1993, 1995; Wilson and Hamilton, 1996] used solar spectrum averaged single scattering properties derived from Viking lander studies by Pollack et al. [1979] at solar wavelength and from Mariner 9 IRIS observations by Toon et al. [1977]. This dust was relatively "dark" (solar averaged single-scattering albedo and asymmetry parameter were 0.86 and 0.79 , respectively) and yielded warm atmospheric temperatures.

[16] After the late 1990s, a second generation of models [Forget et al., 1999; Hartogh et al., 2005; Takahashi et al., 2006] used the improved data set achieved by Ockert-Bell et al. [1997] in the solar range, and by Forget [1998] in the infrared range. It is this data set which is used in the case 1 simulation, and referred to as the Ockert-Bell et al. data set.

[17] Ockert-Bell et al. [1997] extended Viking Lander data corrected by Pollack et al. [1995] to all solar wavelengths $(0.2-4.2 \mu \mathrm{m})$, by using different spectra of bright surfaces under low dust conditions, acquired by the Orbiting Astronomical Observatory in the UV [Wallace et al., 1972] and by Phobos-2 ISM and earth-based telescopes in the visible and near-infrared range [Mustard and Bell, 1994]. The infrared data set $(5-50 \mu \mathrm{m})$ was built on the work by
Toon et al. [1977], who fitted IRIS/Mariner 9 spectra by using a sample of clay called Montmorillonite 219b. This data set was adapted for GCMs by Forget [1998], who removed the $20 \mu \mathrm{m}$ absorptions of this mineral which are not observed on Mars.

[18] These first properties thus merge information from different instruments looking at different locations and times in the solar and thermal domains. Consequently, the dust particle size distributions (which control the balance between dust absorption at solar wavelengths and emission in the infrared region) are different from one observation to another, and thus different for the two domains. Consequently, the ratio of the extinction efficiency (and thus opacity) in the visible to the one in the infrared (later called the "solar over infrared ratio") is specified to correct for this bias and merge both data sets. Here, we use $Q_{\text {ext,GCM }}(0.67 \mu \mathrm{m}) /$ $Q_{\text {ext,GCM}}(9 \mu \mathrm{m})=2$ [Forget, 1998; Toigo and Richardson, 2000]. The resulting single scattering parameters are shown in Figure 1 (grey line), and the corresponding values in the five channels of the GCM are reported in Table 2. These properties are assumed constant in space and time.

[19] It is worth adding that in the time between the retrieval of the aforementioned radiative properties and the second unified data set of Wolff et al. [2006, 2009], different values have been adopted for use in climate models. The measurements of Clancy and Lee [1991] have been used in many GCMs [Forget et al., 1999; Montabone et al., 2006; Richardson et al., 2002; Basu et al., 2004, 2006], but with caution due to the unusually low value of the asymmetry factor $g$. More recently, Hinson and Wilson [2004], Wilson et al. [2007], and Wilson et al. [2008a] have used $\omega_{0}=0.92$ and $g=0.75$ in the GFDL model, and the IR properties derived by Wolff and Clancy [2003].

\subsection{Wolff et al. Model (Case 2 Simulation)}

[20] The second unified data set comes from MGS (Mars Global Surveyor) and MRO (Mars Reconnaissance Orbiter) overflights of the MERs (Martian Exploration Rovers), which enabled the simultaneous observation of dust both from the surface and from space by instruments having similar spectral windows.

[21] Wolff et al. [2006, 2009] combined the "best parts" of each data set in order to effectively isolate the average scattering properties of the suspended dust particles. More specifically, using the total column optical depth and surface reflectance properties from MER with the multiangle, multispectral MGS (TES) and MRO (CRISM) observations, they constructed a self-consistent retrieval algorithm which returned the single scattering albedo and associated refractive indices. At the same time, the combined analyses minimized the typical model uncertainties generally encountered in atmospheric remote sensing analyses, i.e., surface reflectance/emission properties, consistent aerosol scattering with respect to wavelength and particle size, etc. Ultimately, the MER-MGS analyses constrain the 7.5-30 $\mu \mathrm{m}$ region while that for MER-MRO cover 0.26-3 $\mu \mathrm{m}$. Wolff et al. [2009] extend this latter coverage to $4 \mu \mathrm{m}$ through the MEXOMEGA observations of Määttänen et al. [2009].

[22] The resulting refractive indices are used to compute the single scattering parameters of the dust population, which is described by a Gamma size distribution of effective 

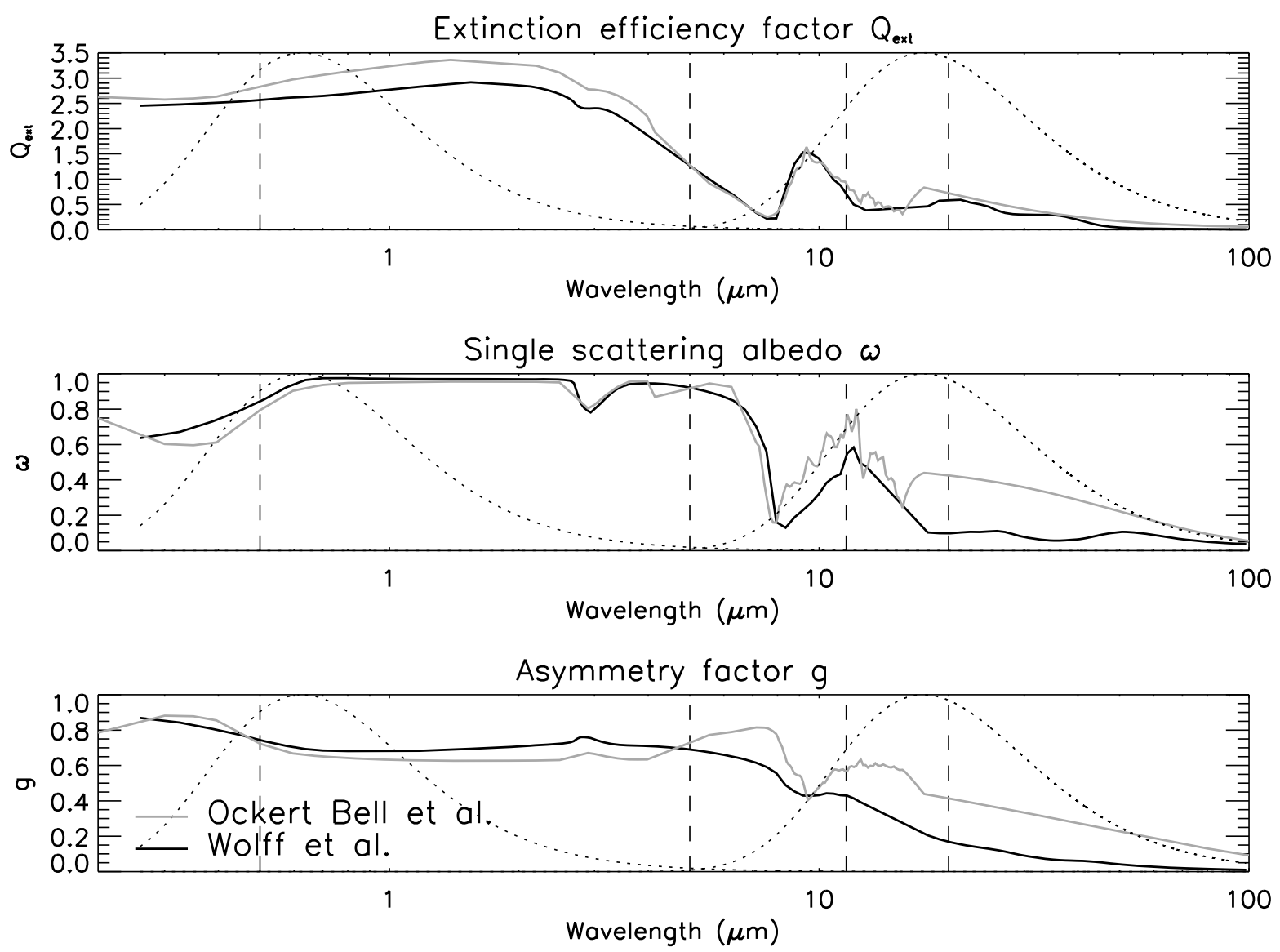

Figure 1. Dust single scattering parameters deduced by Ockert-Bell et al. [1997] and Forget [1998] (grey line) and by Wolff et al. [2006] (black line). (top to bottom) Extinction efficiency factor $Q_{\text {ext }}$, single scattering albedo $\omega_{0}$ and asymmetry parameter $g$. Dashed curves in the background represent the normalized blackbody emission spectra for temperatures of $5870 \mathrm{~K}$ and $210 \mathrm{~K}$, respectively (area preserving representation). Vertical lines separate the 5 channels of the GCM radiative transfer scheme. X-axis is wavelength in micrometers.

radius $\mathrm{r}_{\mathrm{eff}}=1.5 \mu \mathrm{m}$ and variance $\nu_{\mathrm{eff}}=0.3$. Computations are performed by the T-Matrix code of Mishchenko et al. [1996], using cylindrical dust particles and an axial ratio of 1 [Wolff et al., 2001]. Contrary to the case 1 simulation, no assumption needs to be made on the solar over infrared ratio, which depends on the chosen size distribution (here, $\left.Q_{\text {ext,GCM}}(0.67 \mu \mathrm{m}) / Q_{\text {ext,GCM}}(9 \mu \mathrm{m})=1.84\right)$. The computed radiative properties are given in Figure 1 (black line), where they are compared to the Ockert-Bell et al. properties (grey line). The detailed values are reported in Table 2.
[23] Similarly to the case 1 simulation, the case 2 simulation is driven by the untuned TES dust column opacity, and the radiative properties are assumed constant. The dust distribution again follows a modified Conrath profile, and the dust layer depth is still given by an analytical function.

\subsection{TES-Normalized Interactive Dust Model (Case 3 Simulation)}

[24] The last simulation is designed to realistically simulate the vertical distribution and size of the dust particles.

Table 2. Details of the Dust Radiative Properties Used in the Case 1 and 2 Simulations for the Five Channels of the GCM

\begin{tabular}{|c|c|c|c|c|c|c|c|c|c|c|}
\hline \multirow{6}{*}{$\begin{array}{l}\lambda \text { range }(\mu \mathrm{m}) \\
\tilde{Q}_{\text {ext }} / Q_{\text {ext }, 0.67 \mu \mathrm{m}} \\
\tilde{\omega}_{0} \\
\tilde{g}\end{array}$} & \multicolumn{5}{|c|}{ Case 1 Simulation } & \multicolumn{5}{|c|}{ Case 2 Simulation } \\
\hline & \multicolumn{2}{|c|}{ Solar Radiation } & \multicolumn{3}{|c|}{ Thermal Radiation } & \multicolumn{2}{|c|}{ Solar Radiation } & \multicolumn{3}{|c|}{ Thermal Radiation } \\
\hline & $0.1-0.5$ & $0.5-5$ & $5-11.5$ & $11.5-20$ & $20-200$ & $0.1-0.5$ & $0.5-5$ & $5-11.5$ & $11.5-20$ & $20-200$ \\
\hline & 0.878 & 1.024 & 0.406 & 0.253 & 0.166 & 0.952 & 1.024 & 0.382 & 0.175 & 0.120 \\
\hline & 0.665 & 0.928 & 0.541 & 0.470 & 0.370 & 0.738 & 0.955 & 0.348 & 0.285 & 0.0941 \\
\hline & 0.819 & 0.648 & 0.551 & 0.528 & 0.362 & 0.801 & 0.695 & 0.472 & 0.331 & 0.126 \\
\hline
\end{tabular}

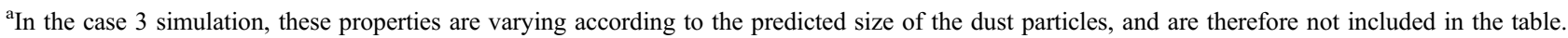
Instead, the solar over infrared ratio $Q_{\text {ext }, 0.67 \mu \mathrm{m}} / Q_{\text {ext,9 }} \mu \mathrm{m}$ is represented in Figure 2. The radiative properties of Ockert-Bell et al. [1997] and Forget [1998] are used in the case 1 simulation, whereas the new properties of Wolff et al. [2006, 2009] are used in the case 2 simulation. The full spectra are shown in Figure 1. The essential difference in $\omega_{0}$ between the two data sets is shown in bold. $\tilde{Q}_{\text {ext }}, \tilde{\omega}_{0}$ and $\tilde{g}$ are computed using the three equations presented at the end of Forget [1998]. 
This allows us to compute the true opacity in each layer (equation (1)) and to take into account the size of the dust particles in the calculation of the single scattering parameters.

\subsubsection{Dust Transport Scheme}

[25] An efficient way to predict the evolution of dust particles is to use a so-called two-moment scheme, used for example by Schulz et al. [1998] to model Saharan dust plumes. If we assume that in any atmospheric layer the size of the dust particles can be described by a lognormal distribution, then three parameters are sufficient to describe the dynamics of the whole particle population. Indeed, the number of particles in each size bin is given in this case by:

$$
n(r)=\frac{N}{\sqrt{2 \pi} \sigma_{0} r} \exp \left[-\frac{1}{2}\left(\frac{\ln \left(r / r_{0}\right)}{\sigma_{0}}\right)^{2}\right],
$$

where $n(r) \mathrm{d} r$ is the number of dust particles per $\mathrm{kg}$ in the size range $[r, r+\mathrm{d} r], N$ the total number of particles per $\mathrm{kg}$, and $r_{0}$ and $\sigma_{0}$ the mean and standard deviation of the distribution. The moments of the lognormal distribution are conveniently expressed by:

$$
M_{k}=\int_{0}^{\infty} r^{k} n(r) \mathrm{d} r=N r_{0}^{k} \exp \left(\frac{k^{2}}{2} \sigma_{0}^{2}\right) .
$$

[26] Consequently, the lognormal distribution is entirely described by $N, r_{0}$ and $\sigma_{0}$. Furthermore, the mass mixing ratio $q$ is directly related to $r_{0}$ by:

$$
\begin{aligned}
q & =\int_{0}^{\infty} \frac{4}{3} \pi r^{3} \rho_{p} n(r) \mathrm{d} r \\
& =\frac{4}{3} \pi \rho_{p} M_{3} \\
& =\frac{4}{3} \pi \rho_{p} N r_{0}^{3} \exp \left(\frac{9}{2} \sigma_{0}^{2}\right) .
\end{aligned}
$$

[27] Therefore, if we assume that the standard deviation $\sigma_{0}$ is constant, the distribution is fully described by the number of particles per $\mathrm{kg} N$ and the mass mixing ratio $q$. The evolution of the dust size distribution can thus be predicted by the GCM using only two tracers. This method is often called a two-moment scheme because $N$ is equal to $M_{0}$ and $q$ is directly related to $M_{3}$. The effective radius of the dust population is then expressed as:

$$
r_{\text {eff }}=\left(\frac{3}{4} \frac{q}{\pi \rho_{p} N}\right)^{1 / 3}\left(1+\nu_{\text {eff }}\right) .
$$

[28] Consequently, dust is represented in the GCM by two tracers, which are injected in the atmosphere by specifying two spatially constant lifting rates $\partial_{t} q$ and $\partial_{t} N$. As reported by Kahre et al. [2008], a spatially and temporally constant lifting rate is sufficient to predict, at least to first order, the spatial distribution of dust particle sizes. The two tracers are advected by a Van Leer scheme I [van Leer, 1977; Hourdin and Armengaud, 1999]. Sedimentation depends on the size of the particles, and the lognormal distribution is discretized into 12 size bins where the sedimentation flux of each tracer is computed. The shape of the particles is accounted for by using the additional $\beta$ factor in the second term of the Cunningham correction factor [see Murphy et al., 1990]. Best results are obtained by setting $\beta$ to 0.5 , as reported by Murphy et al. [1990] when simulating the decay of the 1977A planet-encircling dust storm. The effective radius and variance of the lifted population are set to $3 \mu \mathrm{m}$ and 0.5 respectively, so that the predicted $r_{\text {eff }}$ in the lower levels of the GCM matches the observations of Wolff et al. [2006, Figures 20 and 21].

\subsubsection{Online Single Scattering Parameter Calculation}

[29] The dust particle sizes predicted by the two-moment scheme (see equation (6)) are used to compute, in the GCM, a single scattering parameter field that evolves in space and time. We therefore account for the change in scattering through the dust layer created by differential sedimentation of the dust particles.

[30] To do so, the single scattering parameters for a set of dust particle sizes are computed offline and stored in a lookup table which is loaded by the GCM at the beginning of the simulation. This look-up table contains the single scattering parameters $\mathrm{Q}_{\mathrm{ext}}, \omega_{0}$ and $g$ for 50 sizes of dust particles (from $1 \mathrm{~nm}$ to $10 \mu \mathrm{m}$ ). These single scattering parameters are computed using a lognormal distribution of small effective variance $\left(\nu_{\text {eff }}=0.05\right)$ to remove the ripples created by the interferences in the scattered field [see, e.g., Hansen and Travis, 1974, Figure 8]. Otherwise, these ripples prevent the accurate integration, in the GCM, of the single scattering properties, because of the relatively small number of particle sizes stored in the look-up table. The single scattering parameters are computed from the dust refractive index derived by Wolff et al. [2006, 2009] using the T-Matrix code of Mishchenko et al. [1996], and assuming cylindrical dust particles $(\mathrm{D} / \mathrm{L}=1)$ [Wolff et al., 2001].

[31] The GCM then uses this look-up table and the effective radius predicted by the dust transport scheme to compute in each grid box the integrated single scattering parameters. A 20-point Gauss-Legendre integration is employed, and the results are stored in an interpolation grid to avoid doing the same computation twice if multiple grid boxes contain particles of approximately the same size. A lognormal size distribution of effective variance $\nu_{\text {eff }}=0.3$ is chosen, instead of the Gamma distribution used in the case 2 simulation. The uncertainty on the scattering parameters computed by the GCM for different sizes was determined by comparing them with an optimal code which uses a look-up table of 10000 dust particle sizes. The resulting mean absolute errors for the 5 channels of the GCM (see Table 2) are $\sigma\left(Q_{\text {ext }}\right)=0.04, \sigma\left(\omega_{0}\right)=0.005$ and $\sigma(g)=0.02$. The single scattering parameters vary in space and time, and the same can be said of the solar over infrared ratio. This is illustrated in Figure 2, where the solar over infrared ratio computed over the $L_{s}=210-240^{\circ}$ period is represented for MY25 (panel b) and MY26 (panel d).

\subsubsection{Scaling to the Measured TES Column Opacity}

[32] After calculating the dust sizes, single scattering properties and spatial distribution, the 3D opacity field can be accurately deduced. To do so, the predicted dust mass mixing ratio $q$ (see equation (5)), effective radius $\mathrm{r}_{\text {eff }}$ (see equation (6)) and extinction efficiency factor $Q_{\text {ext }, \lambda}$ (which depends on $\mathrm{r}_{\text {eff }}$ ) are used to compute the opacity in each layer $\mathrm{d} \tau_{\lambda}$ following equation (1). Then, each opacity profile is multiplied by a constant so that the dust column opacity 


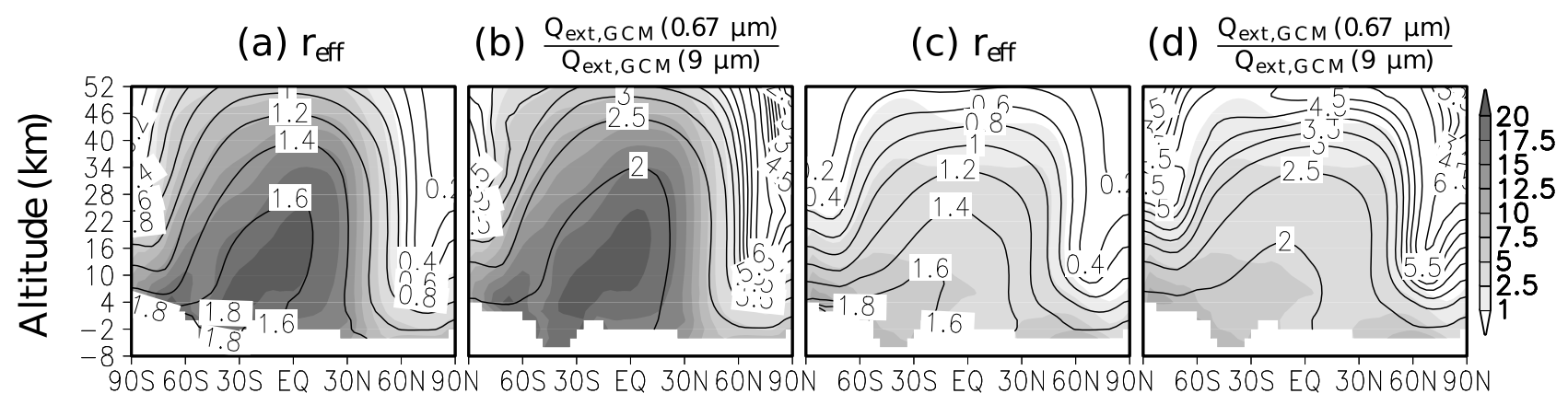

Figure 2. Example of results for the case 3 simulation. (a and c, contours) Solar over infrared ratio. ( $b$ and $\mathrm{d}$, contours) Dust effective radius $\mathrm{r}_{\text {eff }}$ in $\mu \mathrm{m}$. (background shades) Density scaled opacity $g \mathrm{~d} \tau / \mathrm{d} p \times 10^{3}$ at $0.67 \mu \mathrm{m}$. All the fields are averaged zonally and over the $\mathrm{L}_{\mathrm{s}}=210-240^{\circ}$ period. Altitude is given in $\mathrm{km}$ above areoid. The corresponding equatorial mean dust column opacities at $0.67 \mu \mathrm{m}$ are 2.9 and 0.7 for MY25 (left) and MY26 (right), respectively.

matches the TES observations [Smith, 2004]. Consequently, the dust transport model is "semi-interactive," because it explicitly predicts the dust spatial distribution, but uses observations to scale each opacity profile employed in the radiative transfer calculations. Similar "semi-interactive" schemes have been used by Wilson et al. [2008b] in simulations of the 2001 (MY25) global dust storm, and more recently by Kahre et al. [2009] and Wilson [2011].

[33] The resulting density-scaled opacity at $0.67 \mu \mathrm{m}$ is represented in Figure 2 (background shades), along with the effective radius $r_{\text {eff }}$ and computed solar over infrared ratio (contours). The fields are averaged zonally and over the $\mathrm{L}_{\mathrm{s}}=$ 210-240 period for MY25 (panels a and b) and 26 (panels c and d). The density-scaled opacity follows from equation (1) and the hydrostatic equilibrium, and can be written as:

$$
-\frac{1}{\rho_{a}} \frac{\mathrm{d} \tau_{\lambda}}{\mathrm{d} z}=g \frac{\mathrm{d} \tau_{\lambda}}{\mathrm{d} p}=\frac{3}{4} \frac{Q_{\text {ext }, \lambda} q}{\rho_{p} r_{\text {eff }}},
$$

where $\rho_{a}$ is the atmospheric density in $\mathrm{kg} \mathrm{m}^{-3}$. The 2001 global dust storm is clearly visible in panel a, where dust particles of $1.6 \mu \mathrm{m}$ in radius are found near the equator, at an altitude of around $20 \mathrm{~km}$. The dust radiative properties change accordingly, as can be seen by comparing the solar over infrared ratio of MY25 (panel b) with the one of MY26 (panel d). The density-scaled opacity reflects the dynamics of the solsticial Hadley cell, with dust-raising and transport occurring over the southern mid-latitudes.

[34] Dust particle sizes are larger for MY25 than for MY26 because the TES column opacity increases, warms the GCM atmosphere, and feeds back on the Hadley cell intensity. This likely holds true for the $\mathrm{L}_{\mathrm{s}}=210-240^{\circ}$ period represented on Figure 2 which follows the onset of the 2001 dust storm at $\mathrm{L}_{\mathrm{s}}=185^{\circ}$. Consequently, the "semi-interactive" method allows us to capture the main characteristics of the dust layer while remaining constrained by the observed column opacity.

\section{Results Using a Prescribed Dust Vertical Distribution}

[35] In this section, we analyze the temperature biases found in the GCM simulations when the dust distribution is prescribed and the radiative properties are spatially and temporally constant (case 1 and 2 simulations). The analysis will be based on Figures 3, 4, and 5. We will often use the terms "cloud season" and "dust storm season" to refer to the periods $\mathrm{L}_{\mathrm{s}}=40-150^{\circ}$ and $\mathrm{L}_{\mathrm{s}}=180-360^{\circ}$, respectively.

[36] Figures 3 and 4 illustrate the zonal and diurnal mean temperature difference between the GCM simulations and the TES measurements $\left(\Delta \mathrm{T}=\mathrm{T}_{\mathrm{GCM}}-\mathrm{T}_{\mathrm{TES}}\right)$ for MY25 and 26 , respectively. The results are averaged over $30^{\circ}$ of $\mathrm{L}_{\mathrm{s}}$ periods. A positive $\Delta \mathrm{T}$ reflects a warm bias in the GCM simulation. We only focus on the $\pm 50^{\circ}$ latitude band, because the temperature biases in the polar regions are hard to interpret without accounting for the radiative effects of water-ice clouds. Four seasons are represented: one near the aphelion, and three during the dusty perihelion period. The TES diurnal mean temperatures to which the GCM temperatures are compared are given for reference on the left side of Figures 3 and 4 . Figure 5 provides the 2 PM equatorial temperature at the $0.5 \mathrm{hPa}$ pressure level (panel a), along with the dust column opacity at $9.3 \mu \mathrm{m}$ observed by TES (panel b), the dust layer depth (panel c), the effective radius of the dust particles at the $0.5 \mathrm{hPa}$ pressure level (panel d), and the water-ice cloud column opacity at $12.1 \mu \mathrm{m}$ measured by TES (panel e).

[37] The differences in temperature seen in Figures 3, 4, and 5 can come from unresolved dynamical and physical processes, as well as errors in the prediction of the distribution and radiative effect of aerosols.

\subsection{Ockert-Bell et al. Model}

[38] The first simulation uses the dust radiative properties derived by Ockert-Bell et al. [1997] and adapted for the GCM by Forget [1998]. It is worth remembering that these radiative properties are spatially and temporally constant. The temperature differences between this first GCM simulation and the TES data set for MY25 and 26 are summarized in the second column of Figures 3 and 4. During the cloud season (second panel on the first line of Figures 3 and 4), there is a cold bias in the GCM located above the $0.5 \mathrm{hPa}$ pressure level. It is now well established that this cold bias results from the absence of radiatively active water-ice clouds in the GCM [Wilson et al., 2007, 2008a]. Apart from the cold bias due to water-ice clouds, the GCM overestimates temperature by about $10 \mathrm{~K}$, especially during the dust storm 


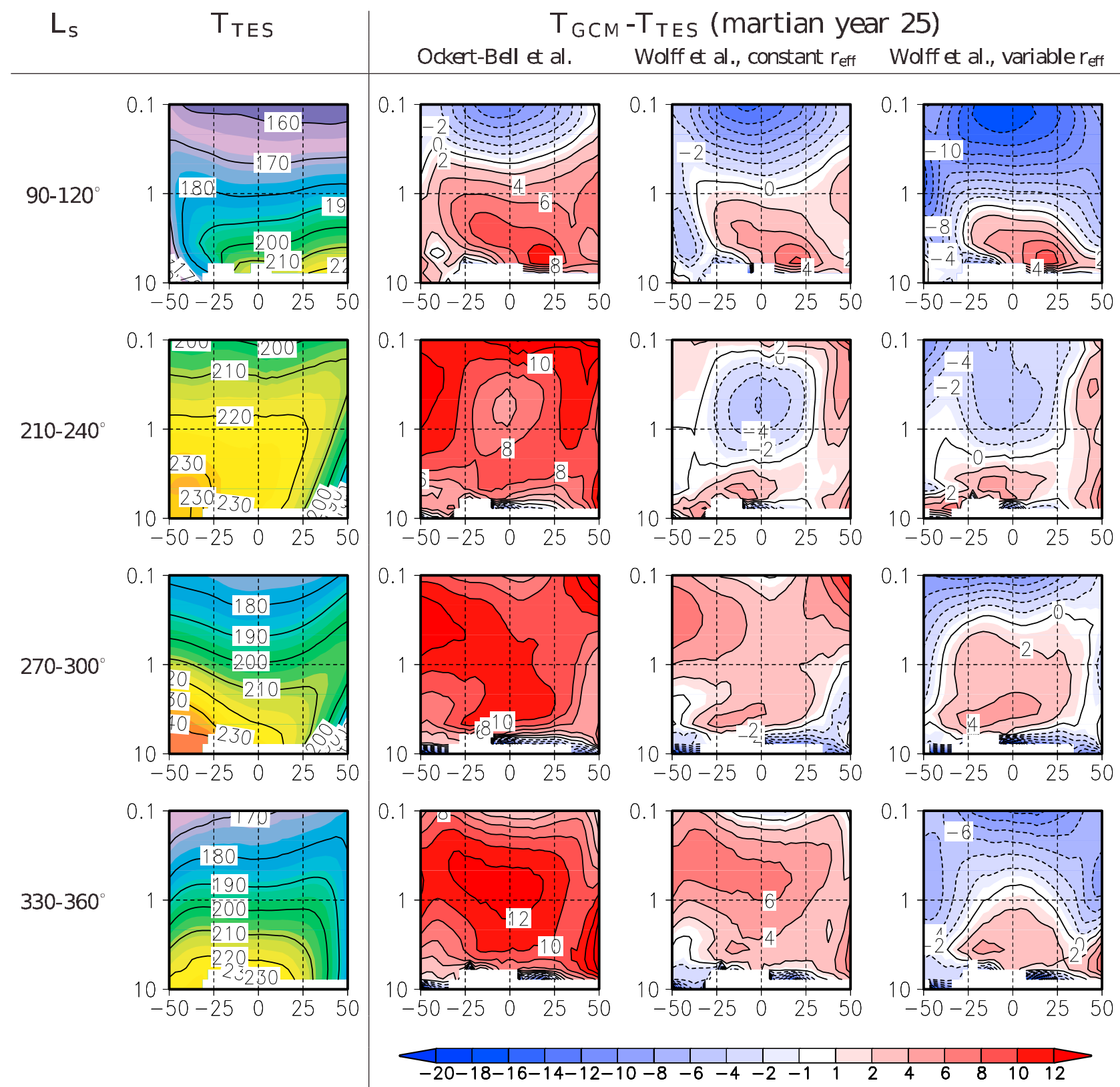

Figure 3. (first column) Zonally and time averaged (over $30^{\circ}$ of $\mathrm{L}_{\mathrm{s}}$ ) temperature fields as measured by TES [Smith, 2002] for Martian year 25. Contour interval is $10 \mathrm{~K}$. (second, third, and fourth columns) Temperature difference between the LMD/GCM and TES averaged over the same time period and for the three reference simulations described in section 2. Contour interval is $2 \mathrm{~K}$. $\mathrm{Y}$-axis is pressure in $\mathrm{hPa}$.

season (see the three last lines in Figures 3 and 4). This is also clearly seen in Figure 5a, where the temperature at the $0.5 \mathrm{hPa}$ pressure level of the case 1 simulation (blue line) is always greater than the observed TES temperature (black crosses). The only exception is the cloud season (see Figure $5 \mathrm{a}$ around $\mathrm{L}_{\mathrm{s}}=90^{\circ}$ ), where the cold bias resulting from the absence of radiatively active water-ice clouds in the GCM is compensated by the overall warm bias.

[39] As we mentioned in the introduction, our knowledge of dust radiative properties has increased in the last decades, and it became apparent that the dust single scattering albedo $\omega_{0}=0.89$ (solar irradiance-weighted average) retrieved by Ockert-Bell et al. [1997] was lower than the values found by
Clancy and Lee [1991] and Clancy et al. [2003] and more recently by Vincendon et al. [2007], Mättänen et al. [2009], and Wolff et al. [2009], which are nearly equal to 0.94 . Consequently, it has been expected that the low value of $\omega_{0}$ will result in overestimated temperatures in climate models [Wolff et al., 2009]. This would explain why there is a general warm bias in the case 1 simulation. In the next section, the radiative properties of dust are updated to test this hypothesis.

\subsection{Effect of the New Wolff et al. Radiative Properties}

[40] The radiative properties are now updated to the most recent data set derived by Wolff et al. [2009] (see Figure 1 


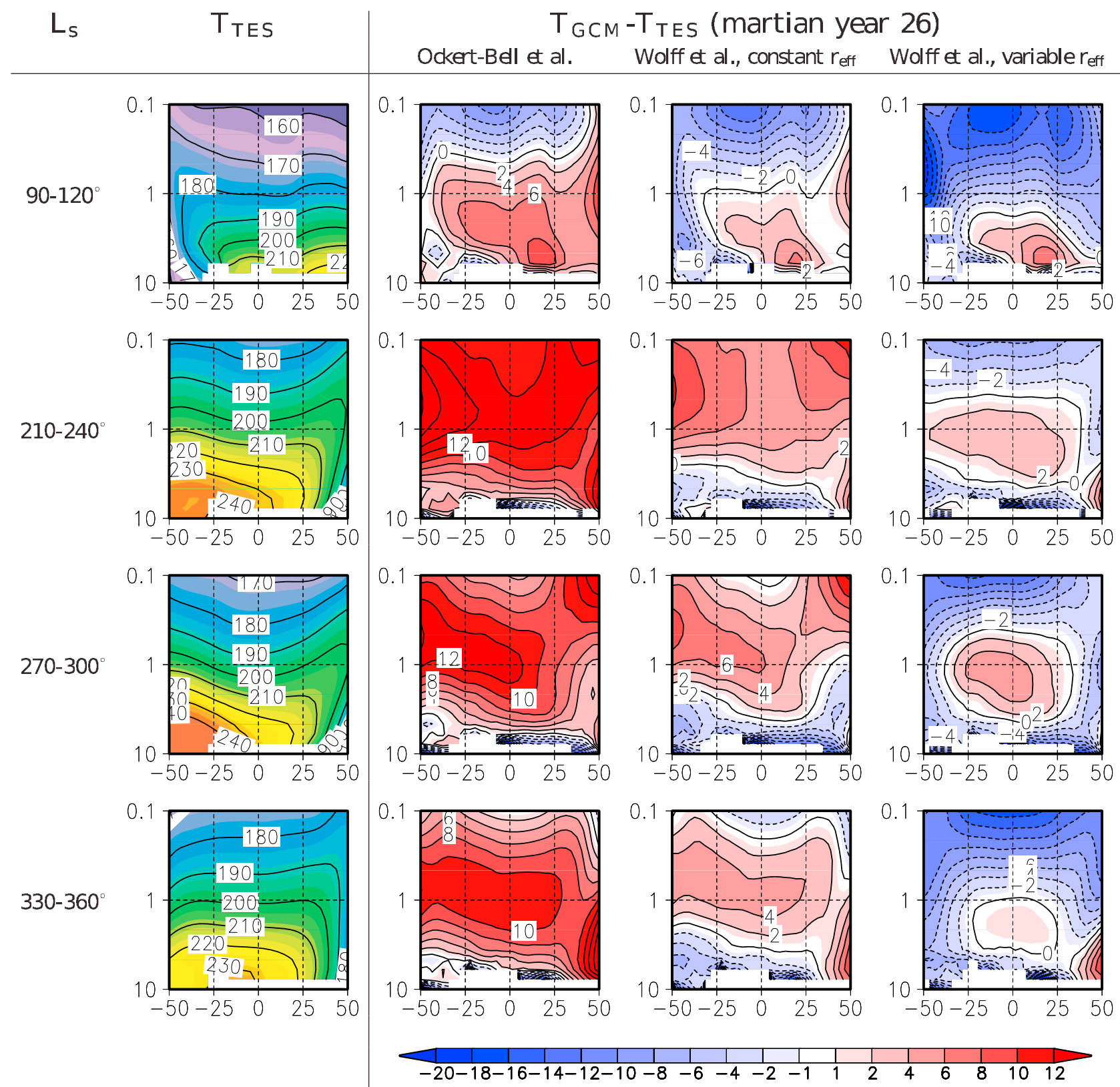

Figure 4. Same as Figure 3 but for Martian year 26.

and Table 2). The main difference between the Ockert-Bell et al. (grey line) and the Wolff et al. (black line) properties lies near the peak of the solar emission spectrum, where the single scattering albedo retrieved by Wolff et al. [2009] is higher than the one measured by Ockert-Bell et al. [1997]. This means that the absorption by dust was probably overestimated in the case 1 simulation. The two $\omega_{0}$ at $0.67 \mu \mathrm{m}$ differ by about $4 \%$ ( 0.970 for the case 2 and 0.929 for the case 1 simulation; see also Table 2), which corresponds to significant changes in the predicted heating rates, as mentioned in the introduction.

[41] The expected cooling effect of the new dust properties is apparent during the dust storm season in Figures 3 and 4 (lines 2 to 4 , third column). The warm bias is reduced by $6-$ $8 \mathrm{~K}$, but the model still overestimates temperature, especially near the $1 \mathrm{hPa}$ pressure level. Consequently, using a brighter dust improves the results, but significant differences remain. [42] The periods of largest temperature anomalies can be identified in Figure 5a, where the temperature at the $0.5 \mathrm{hPa}$ pressure level is represented in green. The model fails to reproduce the temperature variability before and after the peak of the dust storm (right before $\mathrm{L}_{\mathrm{s}}=180^{\circ}$ and at $\mathrm{L}_{\mathrm{s}}=$ $330^{\circ}$ ) for $\mathrm{MY} 25$, and at $\mathrm{L}_{\mathrm{s}}=180^{\circ}$ for MY26, at seasons when the water-ice cloud column opacity is relatively low (see Figure 5e). These differences cannot be attributed to the dust opacity scenario (see Figure $5 b$ ), which presents the same behavior as the measured temperature. For example, before the peak of the 2001 dust storm, at $\mathrm{L}_{\mathrm{s}}=180^{\circ}$, the observed rise in temperature is sudden, as is the case in the dust opacity scenario. Why, then, does the GCM fail in reproducing these sudden temperature increases? 
[43] These biases have also been reported by Wilson et al. 2008a], who noticed a temperature anomaly of 2 to $4 \mathrm{~K}$ for MY24 and 26, and a less pronounced bias for MY25. They attributed these departures from the observed temperatures to variations in the dust layer depth and particle sizes which were not represented in their control simulation, which is also our case. If this hypothesis is correct, we can expect the case 3 simulation to return better results.
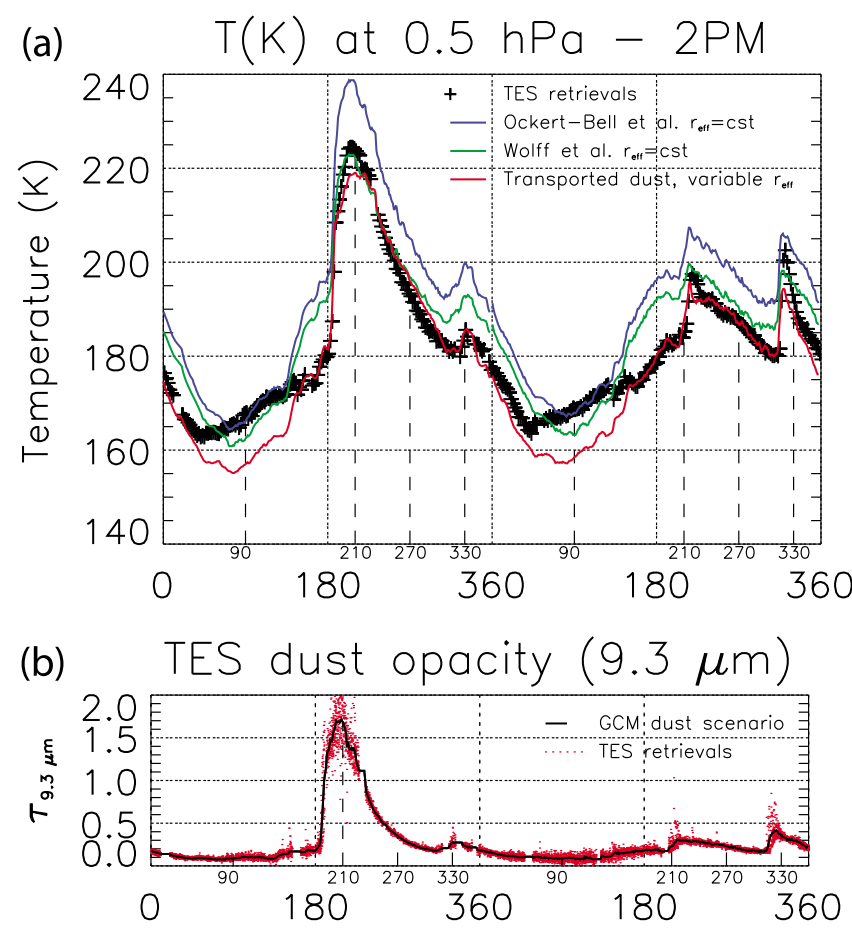

(c) Dust layer top altitude

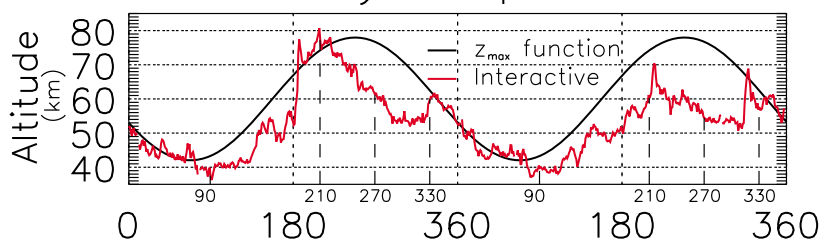

(d) Dust effective radius
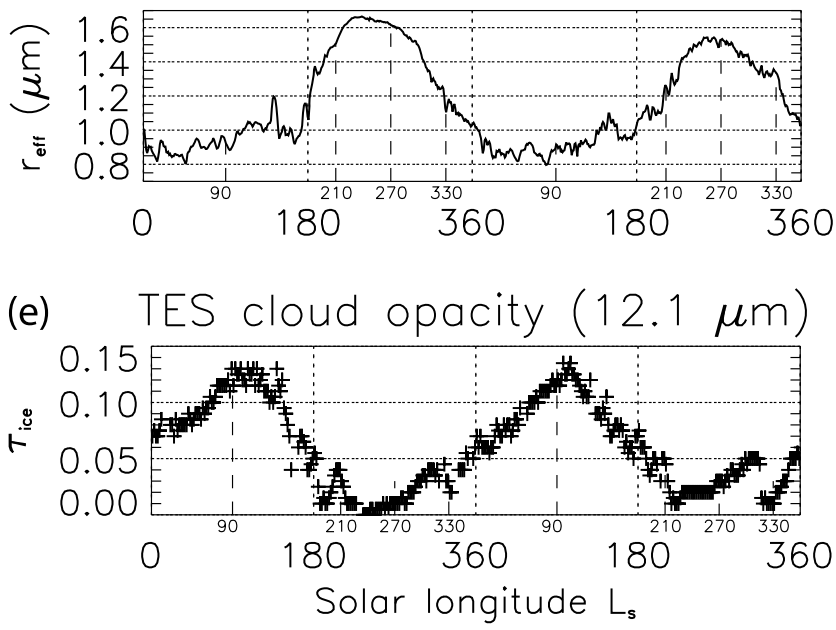

[44] Finally, a cold bias of around $4 \mathrm{~K}$ is found near the $1 \mathrm{hPa}$ pressure level at the peak of the 2001 dust storm (see Figure 3, line 2, third column). There are many possible explanations for this cold bias. Firstly, the dust layer depth might be larger than the specified $Z_{\max }$ (see section 2.1). Secondly, the real dust vertical profile probably differs from the modified Conrath profile used in this simulation (see equation (2)). This is supported by dust transport experiments performed by Kahre et al. [2008], which revealed the formation of an enriched dust layer during storm periods at an altitude that corresponds to our cold bias. Elevated dust layers are also shown by Basu et al. [2006], and Wilson et al. [2008b] also emphasize the inappropriateness of a Conrathtype profile. Recent analysis of MCS observations by Heavens et al. [2011] further discusses this issue. Finally, the real size of the dust particles might also be different from the one used to compute the spatially constant dust radiative properties $\left(\mathrm{r}_{\text {eff }}=1.5 \mu \mathrm{m}\right)$.

[45] These results motivated the development of the case 3 simulation, where the dust vertical distribution and particle size are predicted by the model and taken into account in the radiative calculations. The results of this simulation are analyzed in the next section.

\section{Results of the Semi-Interactive Dust Model}

\subsection{Role of the Dust Layer Depth and Particle Size}

[46] In this last simulation, the dust spatial distribution and radiative properties are varying based on the predictions of the semi-interactive dust model. The results are presented in the last columns of Figures 3 and 4. At pressures higher than $1 \mathrm{hPa}$, the temperatures predicted by the case 2 and case 3 simulations are similar. This is expected because the size distribution of the lifted population was chosen so that the effective radius of the particles in the lower atmosphere is consistent with the measurements of Wolff et al. [2006], as in the case 2 simulation. Moreover, we use the same dust refractive index in both simulations, and the dust layer is often well represented by a modified Conrath profile at these altitudes.

Figure 5. (a) Zonally averaged equatorial temperature at $2 \mathrm{PM}$ for the $0.5 \mathrm{hPa}$ pressure level from TES climatology (crosses, Martian year 25 and 26) [Smith, 2004], and as predicted by the LMD/GCM for three different cases: a simulation using the Ockert-Bell radiative properties (blue curve), another simulation using the Wolff et al. properties (green curve) and a last simulation that uses a semi-interactive dust model (red curve). Vertical dashed lines indicate the four different seasons that are further depicted in Figures 3 and 4 . (b) Dust column opacity at $9.3 \mu \mathrm{m}$ used to guide the model (in black), compared to the raw TES data set at 2 PM (red points). (c) Analytical $z_{\max }$ function used for the case 1 and 2 simulations (black line), compared to the dust layer depth actually predicted by the model in the case 3 simulation (red line). (d) Dust effective radius predicted by the GCM at the $0.5 \mathrm{hPa}$ pressure level for the case 3 simulation. (e) Water-ice cloud column opacity at $12.1 \mu \mathrm{m}$ measured by TES [Smith, 2004]. All the variables are zonally averaged and taken at the equator. 
[47] However, a general cooling is seen in the case 3 simulation when compared to the case 2 simulation, near and above the $1 \mathrm{hPa}$ pressure level. In these regions and during the cloud season, the model temperature can be colder than observed by more than $15 \mathrm{~K}\left(\mathrm{~L}_{\mathrm{s}}=90-120^{\circ}\right.$, first line of Figures 3 and 4, last column). This bias is discussed further in the next section.

[48] During the dust storm season, the warm biases that were present in the case 2 simulation almost disappear. Near the $1 \mathrm{hPa}$ pressure level, the model temperatures are close to the measured values and the warm bias is reduced to $\sim 2 \mathrm{~K}$. The details of the temperature variations at the $0.5 \mathrm{hPa}$ pressure level are represented in Figure 5a. A much better agreement is seen between the model temperature (in red) and the observations (in black), and the only discrepancy occurs during the cloud season, when the observed cloud column opacity at $12.1 \mu \mathrm{m}$ is higher than $\sim 0.07$ (see Figure $5 \mathrm{e}$ ).

[49] As described in section 3.2, the case 2 simulation tends to overestimate temperature, especially before and after the global dust storm of MY25, and near $\mathrm{L}_{\mathrm{s}}=180^{\circ}$ for MY26 (Figure 5a, green curve). In the case 3 simulation, these departures from the observations are corrected (see the red curve in Figure 5a). The sudden rise in temperature before the global dust storm of MY25, as well as the decrease in temperature during the decay of the storm, are well reproduced. Similarly, the model temperature now follows the observations near $\mathrm{L}_{\mathrm{s}}=180^{\circ}$ for MY26. These improvements are mainly due to the improved assessment of the dust layer depth, which is illustrated in Figure $5 \mathrm{c}$ (red line), and compared to the $z_{\max }$ parameter used in the case 1 and 2 simulations (black line). It is seen that the previously prescribed $z_{\max }$ can be larger than the simulated dust layer depth by as much as $20 \mathrm{~km}$, especially when warm biases were seen. The same can be said of the simulated size of the dust particles (Figure 5d) which is most of the time lower than the values used to compute the spatially constant single scattering parameters of the case 1 and 2 simulations.

[50] Consequently, the model was overestimating the amount and size of the dust particles at high altitudes in the case 1 and 2 simulations, thereby absorbing too much solar radiation and overestimating the heating rates. This result is consistent with the early expectations of Wilson et al. [2008a], and with the preliminary radiative experiments performed by Kahre et al. [2008, section 6.5]. Supplementary simulations show that the temperature improvements of the case 3 simulation are mostly due to the better assessment of the dust layer depth, rather than to the changes in radiative properties due to dust particle sizes. Indeed, if the size of the dust particles is kept constant at a value of $1.5 \mu \mathrm{m}$ for the radiative transfer calculations of the case 3 simulation, the temperatures are only modified by about $\pm 1 \mathrm{~K}$ compared to the complete simulation. The impact of varying dust particle sizes on the temperatures is thus secondary compared to that of dust vertical profile. This does not mean, however, that the effect of dust particle size is negligible, and large errors in the prediction of the size of the dust particles lead to a bad fit as well.

\subsection{Remaining Temperature Biases}

\subsubsection{Radiative Effect of Water-Ice Clouds}

[51] Despite the overall improvement provided by the semi-interactive dust model, various differences remain between the model results and the data. The largest bias occurs during the cloud season, where the model temperatures are colder than observed near the $0.1 \mathrm{hPa}$ pressure level. This cold bias is expected, though, because we neglect the radiative effect of water-ice clouds in our simulations. The upper-right panels of Figures 3 and 4 can be compared in this respect to Figure 2b of Wilson et al. [2008a], who investigated the possible origin of this bias and attributed it to the radiative effect of water-ice clouds. Outside the cloud season, this temperature bias is reduced, as can be seen in the upper panel of Figure 5, where the model temperature (in red) only deviates from the observations (in black) between $\mathrm{L}_{\mathrm{s}}=50^{\circ}$ and $\mathrm{L}_{\mathrm{s}}=150^{\circ}$. It is worth noting that since the rest of the model temperature curve fits the observations, an assessment of the radiative heating of water-ice clouds can be made, and is found to reach $10 \mathrm{~K}$.

[52] This cold bias of the model near the $0.1 \mathrm{hPa}$ pressure level is less pronounced during the peak of the 2001 global dust storm (last column and second row of Figure 3), where it is close to $-4 \mathrm{~K}$. It is also relatively weak during MY26 for the same period $\left(\mathrm{L}_{\mathrm{s}}=210-240^{\circ}\right)$. This time period contains the lowest cloud opacities in both years (see Figure 5e). During the rest of the dust storm season, the cold bias can exceed $-10 \mathrm{~K}$, especially during the $\mathrm{L}_{\mathrm{s}}=330-360^{\circ}$ period, during which the aphelion cloud belt starts to form (see the lower-right panel of Figure 4). Therefore, the cold bias seems to vary as a function of water-ice cloud opacity. Moreover, it was also noticed in simulations performed by Basu et al. [2004], who also neglected the radiative effect of water-ice clouds. Consequently, everything points to the fact that the remaining discrepancies mainly come from the neglect of the radiative effect of water-ice clouds.

[53] Preliminary results using the LMD/GCM confirm this hypothesis [Madeleine et al., 2011] and show that this cold bias is significantly reduced when the radiative effect of water-ice clouds is taken into account, including during seasons when the measured water-ice cloud column opacity is relatively low. The significant cold bias seen in the model for the $\mathrm{L}_{\mathrm{s}}=90-120^{\circ}$ period at $50^{\circ} \mathrm{S}$ (see in particular the upper-right panel of Figure 4) is corrected. This results from an increased adiabatic warming in the return branch of the Hadley cell when water-ice clouds are radiatively active [Wilson et al., 2008a; Madeleine et al., 2011]. The detailed impact of radiatively active water-ice clouds on the model temperatures is currently under study, and will be the subject of a future publication.

\subsubsection{Uncertainty in the Dust Distribution and Size}

[54] Another possible source of disagreement between our model and the observations is the uncertainty in the dust spatial distribution and particle size.

[55] First, the semi-interactive dust transport scheme used in the case 3 simulation might fail to predict the regional details of the dust spatial distribution because it relies on the assumption of a spatially uniform lifting rate. Moreover, the size of the dust particles which are injected at the surface is assumed uniform as well, which may also affect the results. For example, the cold bias seen during the 2001 dust storm near the $1 \mathrm{hPa}$ pressure level in the case 2 simulation is unchanged in the case 3 simulation, despite the prediction by the model of a high dust opacity layer at this altitude (see the two left panels of Figure 2). Therefore, the model may not be able to accurately predict the amount and size of the 
dust particles at this altitude, because of the aforementioned reasons. It is also worth noting that recent dust column opacity retrievals by Wilson et al. [2011] suggest that the TES dust column opacities are underestimated during the 2001 global dust storm, which might explain this cold bias as well.

[56] Second, if in future simulations the cold bias at the $0.1 \mathrm{hPa}$ pressure level is not completely corrected by the inclusion of the radiative effect of water-ice clouds, it may also mean that there is more dust in reality than in the model at this altitude, and that the dynamics of the dust layer is not fully captured by the unimodal size distribution employed in our dust transport scheme. A bimodal size distribution of Martian dust particles has been suggested in the past, for example by Montmessin et al. [2002], who proposed the existence of a population of submicron particles to explain an observation of the Viking Orbiter camera. Another population of submicron particles would reach higher altitudes in the model, and warm the layers where the cold bias is present.

[57] Finally, another cold bias also occurs during rapid dust column opacity increases. It can be seen in Figure 5a, during the peak of the 2001 dust storm $\left(\mathrm{L}_{\mathrm{s}}=210^{\circ}\right)$ and also during the two regional dust storm of MY26 $\left(\mathrm{L}_{\mathrm{s}}=220^{\circ}\right.$ and $\mathrm{L}_{\mathrm{s}}=320^{\circ}$ ). During these short periods, the TES temperature increases suddenly, and the model does not reproduce this behavior. As we can see in Figure 5b, there is a dispersal of the TES column opacity values (red dots) during the three periods mentioned above. This variability is smoothed out in the dust opacity scenario of the model (black line), and this might explain why the resulting temperatures are lower than observed. This dispersal of the TES column opacity values also reflects regional disparities in the dust column opacity field due to the preferential lifting of dust particles in certain regions. During storm events, the amount and size of lifted particles strongly depend on the positive feedback between atmospheric dust heating and lifting through changes in wind velocity. This feedback, which has a strongly nonlinear and threshold-linked behavior [Newman et al., 2002; Basu et al., 2004; Kahre et al., 2008], might be responsible for the rapid increase in the observed temperature during the rise of dust storm events and is not captured by our model in which a uniform lifting rate is assumed.

\section{Conclusion}

[58] It has been difficult in the last decade to predict, in the LMD/GCM, a realistic temperature while using at the same time the observed dust column opacity values. Indeed, the model tended to overestimate temperature if the dust column opacity used to drive the model was not appropriately tuned. In this paper, we identify the origin of this disagreement by using the most recently derived dust radiative properties, and we refine our analysis in an attempt to be the closest to the observed TES temperatures. The main results can be summarized as follows:

[59] 1 . The use of the most recently derived dust radiative properties [Wolff et al., 2006, 2009] allows a good prediction of the atmospheric temperatures, while being at the same time consistent with the column opacity measured by TES. The temperature overestimation in previous versions of the GCM was the result of dust being too dark in the
Ockert-Bell et al. [1997] data set. Indeed, the dust single scattering albedo at $0.67 \mu \mathrm{m}$ retrieved by Wolff et al. [2009] is higher by $4 \%$ than the value measured by Ockert-Bell et al. [1997] (see Figure 1, second panel). As illustrated in Figure 5a, the $2 \mathrm{PM}$ temperatures at the $0.5 \mathrm{hPa}$ pressure level are clearly overestimated when using the Ockert-Bell et al. radiative properties (blue line), whereas a good agreement is achieved by using the Wolff et al. properties (green line). However, significant warm biases remain near and above the $1 \mathrm{hPa}$ pressure level, especially before and after dust storm events.

[60] 2. Using a dust transport scheme to account for the spatial distribution and size of the dust particles in radiative transfer calculations removes the above mentioned warm biases (red line in Figure 5a). We show that the dust layer depth prescribed in the previous versions of the model was often too high, resulting in too much absorption of solar radiation by dust in the middle atmosphere. This finding is consistent with the early expectations of Wilson et al. [2008a], and underlines the importance of accurately assessing the dust layer depth in Mars climate models.

[61] 3. In all simulations, a cold bias is persistent near the $0.1 \mathrm{hPa}$ pressure level. Preliminary simulations suggest that this cold bias can be mainly attributed to the neglect of the water-ice cloud radiative effect, especially during the cloud season. Heating by a population of small, high altitude dust particles which are not well represented in the model may contribute to this bias. It would suggest the existence on Mars of another population of submicron particles which is not captured by the unimodal size distribution assumed in our dust transport model. A cold bias is also noticed in the model near the $1 \mathrm{hPa}$ pressure level during the peak of the 2001 dust storm, suggesting that our model fails in simulating the right amount and size of the dust particles under intense storm conditions.

[62] The detailed analysis of the radiative effect of waterice clouds in the LMD/GCM is underway and will allow us to extend the present analysis to the polar regions.

[63] Next steps include the building of new dust opacity scenarios, the modeling of recent Martian years, and the comparison with the Mars Climate Sounder observations, which have already revealed many fascinating processes [McCleese et al., 2008; Kleinböhl et al., 2009; Heavens et al., 2010].

[64] Acknowledgments. We express our gratitude to Aymeric Spiga, Fantine Huot, Jacques Lefrère, Robin Wordsworth, Arnaud Colaitis, Julien Faure, Steve Lewis and Tran The Trung for providing invaluable assistance in developing, testing and validating the model. We also acknowledge constant support from the teams of the UPMC, LMD and IPSL supercomputing facilities (R. Franchisseur, V. Fabart, F. Gueudet, M. Krawczyk, S. Rohrbach, F. Bongat and P. Weill). Very helpful and constructive advice regarding the text was provided by reviewers Claire Newman and John Wilson, and by Pascal Rannou and Peter Read. We also thank Nicolas Mangold and Thierry Fouchet who have provided constant and thoughtful support for this work and the associated thesis. The LMD Mars GCM has been developed with the support of Centre National de la Recherche Scientifique (CNRS), European Space Agency (ESA), and Centre National d'Études Spatiales (CNES) in collaboration with the AOPP (Atmospheric, Oceanic, and Planetary Physics laboratory, Oxford University) and IAA (Instituto de Astrofísica de Andalucía, Granada) groups. Grant support for this work was provided by the UPMC university through doctoral fellowship and by ESA and CNES. Finally, we are indebted to the editorial team of JGR Planets for manuscript preparation and publication. 


\section{References}

Basu, S., M. I. Richardson, and R. J. Wilson (2004), Simulation of the Martian dust cycle with the GFDL Mars GCM, J. Geophys. Res., 109, E11006, doi:10.1029/2004JE002243.

Basu, S., J. Wilson, M. Richardson, and A. Ingersoll (2006), Simulation of spontaneous and variable global dust storms with the GFDL Mars GCM J. Geophys. Res., 111, E09004, doi:10.1029/2005JE002660.

Christensen, P. R., et al. (2001), Mars Global Surveyor Thermal Emission Spectrometer experiment: Investigation description and surface science results, J. Geophys. Res., 106, 23,823-23,872.

Clancy, R. T., and S. W. Lee (1991), A new look at dust and clouds in the Mars atmosphere: Analysis of emission-phase-function sequences from global Viking IRTM observations, Icarus, 93, 135-158.

Clancy, R. T., B. J. Sandor, M. J. Wolff, P. R. Christensen, M. D. Smith, J. C. Pearl, B. J. Conrath, and R. J. Wilson (2000), An intercomparison of ground-based millimeter, MGS TES, and Viking atmospheric temperature measurements: Seasonal and interannual variability of temperatures and dust loading in the global Mars atmosphere, J. Geophys. Res., 105, 9553-9572.

Clancy, R. T., M. J. Wolff, and P. R. Christensen (2003), Mars aerosol studies with the MGS TES emission phase function observations: Optica depths, particle sizes, and ice cloud types versus latitude and solar longitude, J. Geophys. Res., 108(E9), 5098, doi:10.1029/2003JE002058.

Conrath, B. J. (1975), Thermal structure of the Martian atmosphere during the dissipation of the dust storm of 1971, Icarus, 24, 36-46.

Conrath, B. J., J. C. Pearl, M. D. Smith, W. C. Maguire, P. R. Christensen, S. Dason, and M. S. Kaelberer (2000), Mars Global Surveyor Therma Emission Spectrometer (TES) observations: Atmospheric temperatures during aerobraking and science phasing, J. Geophys. Res., 105, 9509-9520.

Dufresne, J.-L., R. Fournier, C. Hourdin, and F. Hourdin (2005), Net exchange reformulation of radiative transfer in the $\mathrm{CO}_{2} 15-\mu \mathrm{m}$ band on Mars., J. Atmos. Sci., 62, 3303-3319.

Forget, F. (1998), Improved optical properties of the Martian atmospheric dust for radiative transfer calculations in the infrared, Geophys. Res. Lett. $25,1105-1108$

Forget, F., F. Hourdin, R. Fournier, C. Hourdin, O. Talagrand, M. Collins, S. R. Lewis, P. L. Read, and J.-P. Huot (1999), Improved general circulation models of the Martian atmosphere from the surface to above 80 km, J. Geophys. Res., 104, 24,155-24,176.

Gierasch, P. J., and R. M. Goody (1972), The effect of dust on the temperature of the Martian atmosphere., J. Atmos. Sci., 29, 400-402.

Haberle, R. M., J. B. Pollack, J. R. Barnes, R. W. Zurek, C. B. Leovy, J. R. Murphy, H. Lee, and J. Schaeffer (1993), Mars atmospheric dynamics as simulated by the NASA AMES General circulation model: 1, The zonal-mean circulation, J. Geophys. Res., 98, 3093-3123.

Haberle, R. M., J. R. Barnes, J. R. Murphy, M. M. Joshi, and J. Schaeffer (1997), Meteorological predictions for the Mars Pathfinder lander, J. Geophys. Res., 102, 13,301-13,312.

Haberle, R. M., et al. (1999), General circulation model simulations of the Mars Pathfinder atmospheric structure investigation/meteorology data J. Geophys. Res., 104, 8957-8974.

Hansen, J. E., and L. D. Travis (1974), Light scattering in planetary atmospheres, Space Sci. Rev., 16, 527-610.

Hartogh, P., A. S. Medvedev, T. Kuroda, R. Saito, G. Villanueva, A. G Feofilov, A. A. Kutepov, and U. Berger (2005), Description and climatology of a new general circulation model of the Martian atmosphere, J. Geophys. Res., 110, E11008, doi:10.1029/2005JE002498.

Heavens, N. G., et al. (2010), Water ice clouds over the Martian tropics during northern summer, Geophys. Res. Lett., 37, L18202, doi:10.1029/ 2010GL044610.

Heavens, N. G., et al. (2011), The vertical distribution of dust in the Martian atmosphere during northern spring and summer: Observations by the Mars Climate Sounder and analysis of zonal average vertical dust profiles, J. Geophys. Res., 116, E04003, doi:10.1029/2010JE003691.

Hinson, D. P., and R. J. Wilson (2004), Temperature inversions, therma tides, and water ice clouds in the Martian tropics, J. Geophys. Res. 109, E01002, doi:10.1029/2003JE002129.

Hourdin, F., and A. Armengaud (1999), The use of finite-volume methods for atmospheric advection of trace species: 1. Test of various formulations in a general circulation model, Monthly Weather Rev., 127(5), 822-837.

Hourdin, F., P. Le van, F. Forget, and O. Talagrand (1993), Meteorological variability and the annual surface pressure cycle on Mars, J. Atmos. Sci. 50, 3625-3640

Hourdin, F., F. Forget, and O. Talagrand (1995), The sensitivity of the Martian surface pressure and atmospheric mass budget to various parameters: A comparison between numerical simulations and Viking observations, J. Geophys. Res., 100, 5501-5523.
Jaquin, F., P. Gierasch, and R. Kahn (1986), The vertical structure of limb hazes in the Martian atmosphere, Icarus, 68, 442-461.

Kahre, M. A., J. L. Hollingsworth, R. M. Haberle, and J. R. Murphy (2008), Investigations of the variability of dust particle sizes in the Martian atmosphere using the NASA Ames general circulation model, Icarus, 195, 576-597.

Kahre, M. A., R. J. Wilson, R. M. Haberle, and J. L. Hollingsworth (2009), An inverse approach to modeling the dust cycle with two Mars general circulation models, in Mars Dust Cycle Workshop.

Kleinböhl, A., et al. (2009), Mars Climate Sounder limb profile retrieval of atmospheric temperature, pressure, and dust and water ice opacity, J. Geophys. Res., 114, E10006, doi:10.1029/2009JE003358.

Määttänen, A., et al. (2009), A study of the properties of a local dust storm with Mars Express OMEGA and PFS data, Icarus, 201, 504-516.

Madeleine, J.-B., F. Forget, and E. Millour (2011), Modeling radiatively active water ice clouds: Impact on the thermal structure and water cycle, paper presented at the Fourth International Workshop on the Mars Atmosphere: Modelling and Observation, CNES, 8-11 February.

McCleese, D. J., et al. (2008), Intense polar temperature inversion in the middle atmosphere on Mars, Nat. Geosci., 1, 745-749.

Mishchenko, M. I., L. D. Travis, and D. W. Mackowski (1996), T-matrix computations of light scattering by nonspherical particles: A review, J. Quant. Spectrosc. Radiat. Transfer, 55, 535-575.

Montabone, L., S. R. Lewis, P. L. Read, and D. P. Hinson (2006), Validation of Martian meteorological data assimilation for MGS/TES using radio occultation measurements, Icarus, 185, 113-132.

Montmessin, F., P. Rannou, and M. Cabane (2002), New insights into Martian dust distribution and water-ice cloud microphysics, J. Geophys. Res., 107(E6), 5037, doi:10.1029/2001JE001520.

Montmessin, F., F. Forget, P. Rannou, M. Cabane, and R. M. Haberle (2004), Origin and role of water ice clouds in the Martian water cycle as inferred from a general circulation model, J. Geophys. Res., 109 , E10004, doi:10.1029/2004JE002284

Murphy, J. R., O. B. Toon, R. M. Haberle, and J. B. Pollack (1990), Numerical simulations of the decay of Martian global dust storms, J. Geophys. Res., 95, 14,629-14,648.

Mustard, J. F., and J. F. Bell (1994), New composite reflectance spectra of Mars from 0.4 to 3.14 micron, Geophys. Res. Lett., 21, 353-356.

Newman, C. E., S. R. Lewis, P. L. Read, and F. Forget (2002), Modeling the Martian dust cycle, 1. Representations of dust transport processes, J. Geophys. Res., 107(E12), 5123, doi:10.1029/2002JE001910.

Ockert-Bell, M. E., J. F. Bell, J. B. Pollack, C. P. McKay, and F. Forget (1997), Absorption and scattering properties of the Martian dust in the solar wavelengths, J. Geophys. Res., 102, 9039-9050.

Pearl, J. C., M. D. Smith, B. J. Conrath, J. L. Bandfield, and P. R. Christensen (2001), Observations of Martian ice clouds by the Mars Global Surveyor Thermal Emission Spectrometer: The first Martian year, J. Geophys. Res., 106, 12,325-12,338

Pollack, J. B., D. S. Colburn, F. M. Flasar, R. Kahn, C. E. Carlston, and D. G. Pidek (1979), Properties and effects of dust particles suspended in the Martian atmosphere, J. Geophys. Res., 84, 2929-2945.

Pollack, J. B., R. M. Haberle, J. Schaeffer, and H. Lee (1990), Simulations of the general circulation of the Martian atmosphere: 1. Polar processes, J. Geophys. Res., 95, 1447-1473.

Pollack, J. B., M. E. Ockert-Bell, and M. K. Shepard (1995), Viking Lander image analysis of Martian atmospheric dust, J. Geophys. Res., $100,5235-5250$.

Richardson, M. I., R. J. Wilson, and A. V. Rodin (2002), Water ice clouds in the Martian atmosphere: General circulation model experiments with a simple cloud scheme, J. Geophys. Res., 107(E9), 5064, doi:10.1029/ 2001JE001804.

Schulz, M., Y. J. Balkanski, W. Guelle, and F. Dulac (1998), Role of aerosol size distribution and source location in a three-dimensional simulation of a Saharan dust episode tested against satellite-derived optical thickness, J. Geophys. Res., 103, 10,579-10,592.

Smith, M. D. (2002), The annual cycle of water vapor on Mars as observed by the Thermal Emission Spectrometer, J. Geophys. Res., 107(E11), 5115, doi:10.1029/2001JE001522.

Smith, M. D. (2004), Interannual variability in TES atmospheric observations of Mars during 1999-2003, Icarus, 167, 148-165.

Smith, M. D., J. C. Pearl, B. J. Conrath, and P. R. Christensen (2000), Mars Global Surveyor Thermal Emission Spectrometer (TES) observations of dust opacity during aerobraking and science phasing, J. Geophys. Res. 105, 9539-9552.

Takahashi, Y. O., H. Fujiwara, and H. Fukunishi (2006), Vertical and latitudinal structure of the migrating diurnal tide in the Martian atmosphere: Numerical investigations, J. Geophys. Res., 111, E01003, doi:10.1029/2005JE002543. 
Toigo, A. D., and M. I. Richardson (2000), Seasonal variation of aerosols in the Martian atmosphere, J. Geophys. Res., 105, 4109-4122.

Toon, O. B., J. B. Pollack, and C. Sagan (1977), Physical properties of the particles composing the Martian dust storm of 1971-1972, Icarus, 30, 663-696.

Toon, O. B., C. P. McKay, T. P. Ackerman, and K. Santhanam (1989), Rapid calculation of radiative heating rates and photodissociation rates in inhomogeneous multiple scattering atmospheres, J. Geophys. Res., 94, 16,287-16,301.

van Leer, B. (1977), Towards the ultimate conservative difference scheme. IV - A new approach to numerical convection, J. Comput. Phys., 23, 276-299.

Vincendon, M., Y. Langevin, F. Poulet, J.-P. Bibring, and B. Gondet (2007), Recovery of surface reflectance spectra and evaluation of the optical depth of aerosols in the near-IR using a Monte Carlo approach: Application to the OMEGA observations of high-latitude regions of Mars, J. Geophys. Res., 112, E08S13, doi:10.1029/2006JE002845.

Wallace, L., J. J. Caldwell, and B. D. Savage (1972), Ultraviolet photometry from the Orbiting Astronomical Observatory: III. Observations of Venus, Mars, Jupiter, and Saturn longward of $2000 \AA$, Astrophys. J., $172,755-769$.

Wilson, R. J. (2011), Dust cycle modeling with the GFDL Mars genera circulation model, paper presented at the Fourth International Workshop on the Mars Atmosphere: Modelling and Observation, CNES, 8-11 February.

Wilson, R. J., and K. Hamilton (1996), Comprehensive model simulation of thermal tides in the Martian atmosphere., J. Atmos. Sci., 53, 1290-1326.

Wilson, R. J., and M. D. Smith (2006), The effects of atmospheric dust on the seasonal variation of Martian surface temperature, paper presented at the Second International Workshop on the Mars Atmosphere: Modelling and Observation, CNES, 27 February to 3 March.

Wilson, R. J., G. A. Neumann, and M. D. Smith (2007), Diurnal variation and radiative influence of Martian water ice clouds, Geophys. Res. Lett. 34, L02710, doi:10.1029/2006GL027976.
Wilson, R. J., S. R. Lewis, L. Montabone, and M. D. Smith (2008a), Influence of water ice clouds on Martian tropical atmospheric temperatures, Geophys. Res. Lett., 35, L07202, doi:10.1029/2007GL032405.

Wilson, R. J., R. M. Haberle, J. Noble, A. F. C. Bridger, J. Schaeffer, J. R. Barnes, and B. A. Cantor (2008b), Simulation of the 2001 planetencircling dust storm with the NASA/NOAA Mars general circulation model, LPI Contrib., 1447,9023.

Wilson, R. J., J. Noble, and S. J. Greybush (2011), The derivation of atmospheric opacity from surface temperature observations, paper presented at the Fourth International Workshop on the Mars Atmosphere: Modelling and Observation, CNES, 8-11 February.

Wolff, M., and R. Clancy (2003), Constraints on the size of Martian aerosols from Thermal Emission Spectrometer observations, J. Geophys. Res., 108(E9), 5097, doi:10.1029/2003JE002057.

Wolff, M. J., R. T. Clancy, K. M. Pitman, J. F. Bell, and P. B. James (2001), Constraints on Martian aerosol particles using MGS/TES and HST data: Shapes, Eos Trans. AGU, 82(47), Fall Meet. Suppl., Abstract P32E-05.

Wolff, M. J., et al. (2006), Constraints on dust aerosols from the Mars Exploration Rovers using MGS overflights and Mini-TES, J. Geophys. Res., 111, E12S17, doi:10.1029/2006JE002786.

Wolff, M. J., M. D. Smith, R. T. Clancy, R. Arvidson, M. Kahre, F. Seelos, S. Murchie, and H. Savijärvi (2009), Wavelength dependence of dust aerosol single scattering albedo as observed by the Compact Reconnaissance Imaging Spectrometer, J. Geophys. Res., 114, E00D04, doi:10.1029/2009JE003350.

F. Forget, E. Millour, and L. Montabone, Laboratoire de Météorologie Dynamique, CNRS/UPMC/IPSL, 4 Pl. Jussieu, BP99, F-75252, Paris CEDEX 05, France.

J.-B. Madeleine, Department of Geological Sciences, Brown University, 324 Brook St., Box 1846, Providence, RI 02912, USA. (jean-baptiste madeleine@brown.edu)

M. J. Wolff, Space Science Institute, 4750 Walnut St., Ste. 205, Boulder, CO 80301, USA. 